\title{
Improved Receding Horizon Fourier Analysis for Quasi-periodic Signals
}

\author{
Bo-Kyu Kwon*, Soohee Han ${ }^{\dagger}$ and Sekyung Han**
}

\begin{abstract}
In this paper, an efficient short-time Fourier analysis method for the quasi-periodic signals is proposed via an optimal fixed-lag finite impulse response (FIR) smoother approach using a receding horizon scheme. In order to deal with time-varying Fourier coefficients (FCs) of quasi-periodic signals, a state space model including FCs as state variables is augmented with the variants of FCs. Through an optimal fixed-lag FIR smoother, FCs and their increments are estimated simultaneously and combined to produce final estimates. A lag size of the optimal fixed-lag FIR smoother is chosen to minimize the estimation error. Since the proposed estimation scheme carries out the correction process with the estimated variants of FCs, it is highly probable that the smaller estimation error is achieved compared with existing approaches not making use of such a process. It is shown through numerical simulation that the proposed scheme has better tracking ability for estimating time-varying FCs compared with existing ones.
\end{abstract}

Keywords: Receding horizon, Fourier analysis, Optimal estimation, Fixed-lag FIR smoother, Timevarying fourier coefficients, Quasi-periodic signal

\section{Introduction}

The problem of estimating the time-varying Fourier coefficients (FCs) of the quasi-periodic signals has been one of the major topics in estimation theories and digital signal processing areas such as speech recognition, energy processing systems, mechanical systems, and general time series analysis $[12,13,16]$.

Under the assumption that the FCs of quasi-periodic signals vary slowly enough to be treated as constants in short-time interval, so called short time Fourier analysis has been developed and improved with the modified or upgraded versions $[14,15]$. As old but practical schemes, the discrete Fourier transform(DFT) with window functions and the recursive discrete Fourier transform(RDFT) methods were proposed to effectively estimate the time-varying FCs of quasi-periodic signals $[1,2,3,4]$. The DFT and RDFT methods can give exact estimations of FCs for a noiseless signal.

However, in most cases, real signals are corrupted with noises and hence the algorithms for estimating FCs should consider its stochastic information for enhancing the performance. In order to avoid the poor performance due to noises, Kalman filtering approaches were suggested in [5, 17]. In these approaches, FCs are included in parts of the state variables, which are estimated from Kalman filter using the stochastic information on noises.

Instead of using the Kalman filter that has infinite

$\dagger$ Corresponding Author: Dept. of Creative IT Engineering, POSTECH, Korea. (sooheehan@postech.ac.kr)

* Dept. of Control \& Instrumentation Engineering, Kangwon National University, Korea. (bkkwon@kangwon.ac.kr)

** Dept. of Electrical Engineering, Kyungpook National University, Korea. (skhan@knu.ac.kr)

Received: March 29, 2016; Accepted: June 28, 2016 impulse response (IIR) structure, the finite impulse response (FIR) structure filter was also tried to be applied to estimate FCs. It has been well acknowledged in signal processing areas and estimation theories that FIR structure tends to be more robust and has the faster response, and hence FIR filters have been more commonly employed compared with IIR filters $[9,10,11]$. In order to employ such good properties of FIR structure, the optimal FIR filter approach was suggested in [6]. Compared with the DFT and RDFT methods for deterministic signals without noises, the state estimation schemes of Kalman IIR filters and FIR filters mentioned above turned out to be effective methods for suppressing noises and achieving fast tracking ability in estimating FCs.

However, existing state space models employed in the Kalman IIR filters and FIR filters for estimating FCs have some limitation of representing the time-varying FCs. Since the zero mean Gaussian random process is employed to represent the variants of time-varying FCs, the tracking ability becomes poor or the convergence speed becomes slow when FCs vary with bias, or increases or decreases monotonically with time. In this regard, it would be very useful to develop the state space model based estimation schemes that can deal with time-varying FCs in a more effective way.

In this paper, we propose a new state space model, where the variants of time varying FCs are considered as parts of state variables and augmented with existing state space models. The estimated variants of time-varying FCs play a role in compensating estimation errors due to time varying FCs and hence achieving the fast convergence to real FCs. While existing approaches assume that FCs vary with time according to an unbiased random walk model, the proposed scheme estimates the variants of FCs directly 
and hence allows for biased random walk models in real situations. In addition, the paper employs more general optimal fixed-lag FIR smoother instead of optimal FIR filter. The optimal fixed-lag FIR smoother can give more general solution than the optimal FIR filter since the optimal FIR smoother is reduced to the FIR filter if the fixed-lag size is set to be zero. Additionally, we show that a fixed-lag size has an effect on the estimation errors. A fixed-lag size of the optimal fixed-lag FIR smoother is chosen to minimize the estimation error variance.

This paper is organized as follows: In Section 2, a new state space model for quasi-periodic signals is introduced to estimate FCs in a more efficient way. In Section 3, a short-time Fourier analysis method is suggested via the optimal fixed-lag FIR smoother. In Section 4, the performances of the proposed scheme and the existing one are compared through a numerical example. Finally, our conclusion is presented in Section 5.

\section{A New State Space Model for Quasi-periodic Signals}

Consider the following quasi-periodic signal model corrupted with measurement noises:

$$
\begin{aligned}
z_{k}= & \frac{a_{0, k}}{\sqrt{2}}+\frac{b_{0, k}(-1)^{k}}{\sqrt{2}} \\
& +\sum_{m=1}^{M-1}\left[a_{m, k} \cos \frac{2 \pi m k}{T}+b_{m, k} \sin \frac{2 \pi m k}{T}\right]+v_{k},
\end{aligned}
$$

where $T$ is the period of the signal and $M$ is the number of harmonic components present in $z_{k}$. We assume that the period $T$ is fixed and known, and $M$ is set to be $T / 2$. Here, $\left\{a_{m, k}, b_{m, k} ; m=1,2, \cdots, M-1\right\}$ are the FCs of the $m$-th harmonic component at time $k$ and they are time varying. $v_{k}$ is the measurement noise, which is a zeromean white Gaussian random process with the covariance $R$. Then, the quasi-periodic signal model in (1) can be represented with the following state space model $[5,6]$ :

$$
\begin{gathered}
x_{k+1}=A x_{k}, \\
z_{k}=C x_{k}+v_{k},
\end{gathered}
$$

where the state variable $x_{k} \in \mathfrak{R}^{2 M \times 1}$ is given by

$$
\begin{aligned}
& x_{k}=\left[\begin{array}{lllllll}
x_{1, k}^{T} & x_{2, k}^{T} & x_{3, k}^{T} & x_{4, k}^{T} & \cdots & x_{2 M-1, k}^{T} & x_{2 M, k}^{T}
\end{array}\right]^{T} \\
& =\left[\begin{array}{c}
a_{0, k} \\
(-1)^{k} b_{0, k} \\
a_{1, k} \cos k \theta+b_{1, k} \sin k \theta \\
-a_{1, k} \sin k \theta+b_{1, k} \cos k \theta \\
\vdots \\
a_{M-1, k} \cos (M-1) k \theta+b_{M-1, k} \sin (M-1) k \theta \\
-a_{M-1, k} \sin (M-1) k \theta+b_{M-1, k} \cos (M-1) k \theta
\end{array}\right]
\end{aligned}
$$

and the system matrix $A \in \mathfrak{R}^{2 M \times 2 M}$ and the measurement matrix $C \in \mathfrak{R}^{1 \times 2 M}$ are given in the following form:

$$
\begin{aligned}
& A=\left[1 \oplus(-1) \oplus \operatorname{bdiag}\left(\begin{array}{cc}
\cos (m \theta) & \sin (m \theta) \\
-\sin (m \theta) & \cos (m \theta)
\end{array}\right)\right], \\
& C=\left[\begin{array}{lllllllll}
\frac{1}{\sqrt{2}} & \frac{1}{\sqrt{2}} & 1 & 0 & 1 & 0 & \cdots & 1 & 0
\end{array}\right] \text {, }
\end{aligned}
$$

where the notations $\oplus$ and bdiag mean the block diagonal matrix expansion operator and the block diagonal matrix composed of its parameters, respectively, and $\theta=2 \pi T$. If it is assumed that the variants of FCs of the $m$-th harmonic components, i.e. $\left\{\Delta a_{m, k}, \Delta b_{m, k} ; m=1,2, \cdots, M-1\right\}$, follow a random walk motion and then the time-varying FCs are represented as

$$
\begin{aligned}
& a_{m, k+1}=a_{m, k}+\Delta a_{m, k} \\
& b_{m, k+1}=b_{m, k}+\Delta b_{m, k}
\end{aligned}
$$

the system model (2)- (3) can be written as

$$
\begin{gathered}
x_{k+1}=A x_{k}+w_{k}, \\
z_{k}=C x_{k}+v_{k},
\end{gathered}
$$

where the system noise $w_{k} \in \mathfrak{R}^{2 M \times 1}$ is defined by

$$
\begin{aligned}
& w_{k}=G_{k} \Delta F_{F, k} \\
& G_{k}=\left[1 \oplus(-1)^{k+1} \oplus\right. \text { bdiag } \\
& \left(\begin{array}{cc}
\cos \{m(k+1) \theta\} & \sin \{m(k+1) \theta\} \\
-\sin \{m(k+1) \theta\} & \cos \{m(k+1) \theta\}
\end{array}\right] \in \mathfrak{R}^{2 M \times 2 M}, \\
& \Delta_{F, k}=\left[\begin{array}{c}
\Delta a_{0, k} \\
\Delta b_{0, k} \\
\vdots \\
\Delta a_{M-1, k} \\
\Delta b_{M-1, k}
\end{array}\right] \in \mathfrak{R}^{2 M \times 1} .
\end{aligned}
$$

In $[5,6]$, the harmonic pair $\Delta a_{m, k}$ and $\Delta b_{m, k}$ were considered as zero mean stationary noises and hence the time invariant variance $Q$ of $w_{k}$ was computed to be

$$
Q=\left[\sigma_{a_{0}} \oplus \sigma_{b_{0}} \oplus \operatorname{bdiag}\left(\begin{array}{cc}
\sigma_{a_{m}} & 0 \\
0 & \sigma_{b_{m}}
\end{array}\right)\right] \in \Re^{2 M \times 2 M},
$$

where $\sigma_{a_{m}}$ and $\sigma_{b_{m}}$ are the covariance of $a_{m}$ and $b_{m}$, respectively.

The state space model (8)-(9) can represent the time varying FCs of the quasi-periodic signal models (1) by reflecting their variants in zero mean and stationary noise 
$w_{k}$. However, it is not practical to assume that the variants of FCs are zero mean stationary noises. In reality, the FCs may vary with bias, or decrease or increase monotonically with time. Additionally, the FCs may not be stationary, which means that $Q$ in (13) may not be constant. We need a more practical noise model for representing time-varying FCs in a more effective way. Therefore, we propose a new state space model for the quasi-periodic signals with timevarying FCs by augmenting the variants of FCs.

To begin with, we define $\Delta x_{k} \in \mathfrak{R}^{2 M \times 1}$ to represent the variants of time varying FCs at time $k$ by replacing $a_{m, k}$ and $b_{m, k}$ with $\Delta a_{m, k}$ and $\Delta b_{m, k}$, respectively, in the definition of $x_{k}$.

By using the variants of time varying FCs $\Delta x_{k}$, the state space model (8)-(9) can be represented as follows.

$$
\begin{gathered}
x_{k+1}=A\left(x_{k}+\Delta x_{k}\right), \\
z_{k}=C x_{k}+v_{k},
\end{gathered}
$$

It is noted that $w_{k}$ in (8) is replaced with $A \Delta x_{k}$ in (14). The variants $\Delta x_{k}$ will be estimated and then combined with $x_{k}$ to generate the final estimates of FCs. Instead of using $w_{k}$ that have effects on FCs directly as in (8), we propose a following noise model

$$
\Delta x_{k+1}=A \Delta x_{k}+\Delta w_{k},
$$

where $\Delta w_{k} \in \mathfrak{R}^{2 M \times 1}$ is zero-mean white Gaussian random process noise with covariance $\bar{Q} \in \mathfrak{R}^{2 M \times 2 M}$. It can be seen that $\Delta w_{k}$ contributes the variants of $\Delta x_{i}$ from $i=k$ to $i=k+1$. If FCs are increased or decreased at the constant rate, $\Delta w_{k}$ would be zero. However, the state space model (8) would create nonzero biased $w_{k}$ to describe timevarying FCs, which means that the time invariant properties of noises does not hold anymore.

By augmenting the state variable with $\Delta x_{k}$, we have

$$
\begin{aligned}
{\left[\begin{array}{c}
x_{k+1} \\
\Delta x_{k+1}
\end{array}\right] } & =\left[\begin{array}{cc}
A & A \\
0 & A
\end{array}\right]\left[\begin{array}{c}
x_{k} \\
\Delta x_{k}
\end{array}\right]+\left[\begin{array}{l}
0 \\
I
\end{array}\right] \Delta w_{k}, \\
z_{k} & =\left[\begin{array}{ll}
C & 0
\end{array}\right]\left[\begin{array}{c}
x_{k} \\
\Delta x_{k}
\end{array}\right]+v_{k},
\end{aligned}
$$

where $I \in \mathfrak{R}^{2 M \times 2 M}$ is an identity matrix.

The observability condition of the augmented system (17)-(18) can be easily checked as follows. If an original system (8)-(9) is observable, the following condition is satisfied:

$$
\operatorname{rank}\left[\begin{array}{c}
C \\
\lambda I-A
\end{array}\right]=2 M
$$

for all $\lambda$. From (19), we have

$$
\operatorname{rank}\left[\begin{array}{cc}
C & 0 \\
\lambda I-A & -A \\
0 & \lambda I-A
\end{array}\right]=4 M
$$

The observability condition (20) is easily proved and its proof is omitted.

To be summarized, the proposed model (17)-(18) is effective in dealing with deterministic components of timevarying FCs. For example, monotonically increasing or decreasing FCs can be properly modeled with (17)-(18).

However, such practically time-varying FCs is not well described with the existing model (8)-(9). For this reason, the proposed model provides the faster tracking performance than the existing one.

\section{Improved Short-time Fourier Analysis using Optimal Fixed-lag FIR Smoother}

Let $\chi_{k}, \bar{A}, \bar{G}$, and $\bar{C}$ as

$$
\begin{aligned}
& \chi_{k}=\left[\begin{array}{c}
x_{k} \\
\Delta x_{k}
\end{array}\right] \in \Re^{4 M \times 1}, \bar{A}=\left[\begin{array}{cc}
A & A \\
0 & A
\end{array}\right] \in \Re^{4 M \times 4 M}, \\
& \bar{G}=\left[\begin{array}{l}
0 \\
I
\end{array}\right] \in \mathfrak{R}^{4 M \times 2 M}, \bar{C}=\left[\begin{array}{ll}
C & 0
\end{array}\right] \in \mathfrak{R}^{1 \times 4 M},
\end{aligned}
$$

the system model (17)-(18) can be rewritten as,

$$
\begin{gathered}
\chi_{k+1}=\bar{A} \chi_{k}+\bar{G} \Delta w_{k}, \\
z_{k}=\bar{C} \chi_{k}+v_{k},
\end{gathered}
$$

For the state space model (22)-(23), the optimal fixed lag FIR smoother can be expressed as a linear function of the finite measurements on the horizon $[k-N, k]$ as

$$
\hat{\chi}_{k-h \mid k}=\left[\begin{array}{c}
\hat{x}_{k-h \mid k} \\
\Delta \hat{x}_{k-h \mid k}
\end{array}\right]=H Z_{k-1} \in \Re^{4 M \times 1},
$$

where $N$ and $h(0<h<N)$ are the horizon length and the fixed-lag size, respectively, and $Z_{k-1}$ is the most recent finite number of measurements on the horizon $[k-N, k]$ as

$$
Z_{k-1}=\left[\begin{array}{llll}
z_{k-N}^{T} & z_{k-N+1}^{T} & \cdots & z_{k-1}^{T}
\end{array}\right]^{T} \in \Re^{N \times 1}
$$

The optimal gain matrix of the fixed lag FIR smoother $H$ for the following minimum error variance criterion,

$$
\begin{aligned}
& \min \mathrm{E}\left[\left(\chi_{k-h}-\hat{\chi}_{k-h \mid k}\right)^{T}\left(\chi_{k-h}-\hat{\chi}_{k-h \mid k}\right)\right] \\
& \text { subject to } \\
& \mathrm{E}\left[\chi_{k-h}\right]=\mathrm{E}\left[\hat{\chi}_{k-h \mid k}\right]
\end{aligned}
$$


is determined by [7]:

$$
\begin{aligned}
H & =\left(\bar{A}^{N-h}-M_{N} Q_{N} \tilde{G}_{N}^{T} \Pi_{N}^{-1} \tilde{C}_{N}\right)\left(\tilde{C}_{N}^{T} \Pi_{N}^{-1} \tilde{C}_{N}\right)^{-1} \\
& \times \tilde{C}_{N}^{T} \Pi_{N}^{-1}+M_{N} Q_{N} \tilde{G}_{N}^{T} \Pi_{N}^{-1} \in \Re^{4 M \times N},
\end{aligned}
$$

where $M_{N}, \Pi_{N}, \tilde{C}_{N}$, and $\tilde{G}_{N}$ are obtained from:

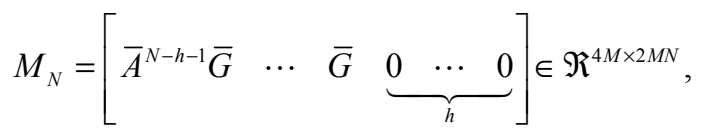

$$
\begin{aligned}
& \Pi_{N}=\tilde{G}_{N} Q_{N} \tilde{G}_{N}^{T}+R_{N} \in \mathfrak{R}^{N \times N}, \\
& \tilde{C}_{N}=\left[\begin{array}{c}
\bar{C} \\
\overline{C A} \\
\vdots \\
\overline{C A}^{N-1}
\end{array}\right] \in \Re^{N \times 4 M}, \\
& \tilde{G}_{N}=\left[\begin{array}{ccccc}
0 & 0 & \cdots & 0 & 0 \\
\bar{C} \bar{G} & 0 & \cdots & 0 & 0 \\
\bar{C} \bar{G} \bar{G} & \bar{C} \bar{G} & \cdots & 0 & 0 \\
\vdots & \vdots & \ddots & \vdots & \vdots \\
\overline{C A}^{N-2} \bar{G} & \bar{C}^{N-3} \bar{G} & \cdots & \bar{C} \bar{G} & 0
\end{array}\right] \in \Re^{N \times 2 M N}
\end{aligned}
$$

and $Q_{N}$ and $R_{N}$ are the diagonal matrices of $Q$ and $R$, respectively, given by:

$$
\begin{gathered}
Q_{N}=[\underbrace{Q \oplus Q \oplus \cdots \oplus Q}_{N}] \in \Re^{2 M N \times 2 M N}, \\
R_{N}=[\underbrace{R \oplus R \oplus \cdots \oplus R}_{N}] \in \Re^{N \times N} .
\end{gathered}
$$

Then, the time varying FCs and their increments can be obtained as follows:

$$
\begin{aligned}
& \hat{a}_{m, k-h \mid k}=\hat{x}_{2 m+1, k-h \mid k} \cos (m \theta k)-\hat{x}_{2 m+2, k-h \mid k} \sin (m \theta k), \\
& \hat{b}_{m, k-h \mid k}=\hat{x}_{2 m+1, k-h \mid k} \sin (m \theta k)-\hat{x}_{2 m+2, k-h \mid k} \cos (m \theta k), \\
& \Delta \hat{a}_{m, k-h \mid k}=\Delta \hat{x}_{2 m+1, k-h \mid k} \cos (m \theta k)-\Delta \hat{x}_{2 m+2, k-h \mid k} \sin (m \theta k), \\
& \Delta \hat{b}_{m, k-h \mid k}=\Delta \hat{x}_{2 m+1, k-h \mid k} \sin (m \theta k)-\Delta \hat{x}_{2 m+2, k-h \mid k} \cos (m \theta k) .
\end{aligned}
$$

Since FIR type estimator is used, the proposed algorithm guarantee the bounded input bounded output (BIBO) stability and the robustness to numerical errors. Moreover, the proposed algorithm could give better estimation than the optimal FIR filtering method because generally the fixed lag smoothers give more accurate estimation than filters. It will be shown through simulation that the proposed method has much better performance than previous filtering approaches. Moreover, the proposed algorithm gives more general solution than the optimal FIR filtering approach in [6], because the optimal fixed-lag smoother with zero lag size is same as the optimal FIR filter. And, as seen in (24), the proposed method does not use any priori information of the state and any artificial assumptions, whereas the optimal FIR filtering approach in [6] use the heuristic infinite covariance of the initial state information.

\section{Numerical Example}

In this section, numerical examples are represented to demonstrate the capabilities of the proposed new method. In order to compare the performance of the proposed method with that of other methods proposed in [6], the test signal is considered as

$$
z_{k}=c_{k} \cos \left(\frac{\pi k}{6}\right)+d_{k} \cos \left(\frac{\pi k}{3}\right)+v_{k},
$$

where magnitudes of the harmonic components $c_{k}$ and $d_{k}$ are taken as

$$
\begin{gathered}
c_{k}=\left\{\begin{array}{ccc}
k / 20 & \text { for } & 0 \leq k<250 \\
k / 2-112.5 & \text { for } & 250 \leq k<500
\end{array},\right. \\
d_{k}=\left\{\begin{array}{ccc}
-k / 20+6 & \text { for } & 0 \leq k<250 \\
-k / 10+18.5 & \text { for } & 250 \leq k<500
\end{array}\right.
\end{gathered}
$$

, respectively.

For the state space model, the system noise covariance $Q$ and the measurement noise covariance $R$ are taken as $I$ and 1 , respectively. The number of harmonic components $M$ is considered as 6 .

In this simulation, we estimate the magnitude of the lower harmonic component at $\pi / 6$, i.e. $c_{k}$, by using the optimal fixed-lag FIR smoother with the proposed new state model and it is compared with the estimates of the optimal FIR filter and fixed-gain Kalman filter with the previous state model in [6]. To design the optimal FIR filter and smoother, the horizon size $N$ and the fixed-lag size $h$ are taken as 12 and 6, respectively. For the fixed-gain Kalman filter design, $\mathcal{E}$ in [6] is set as 1 .

In Fig. 1, the estimation errors $\left(c_{k}\right)$ of the optimal fixedlag FIR smoother with the proposed new state space model (22)-(23) are compared with those of the optimal FIR filter and fixed-gain Kalman filter with the previous state space model in [6].

As shown in the figure, the proposed approach gives a better harmonic estimate for the time-varying FCs, compared with other two previous approaches. As expected, the proposed method gives unbiased estimate whereas estimates of other two approaches are biased, which comes from the assumption that the variants of time-varying FCs are zero mean Gaussian random process. Therefore, it can be said that the proposed method has better estimation performance than the previous approach. 


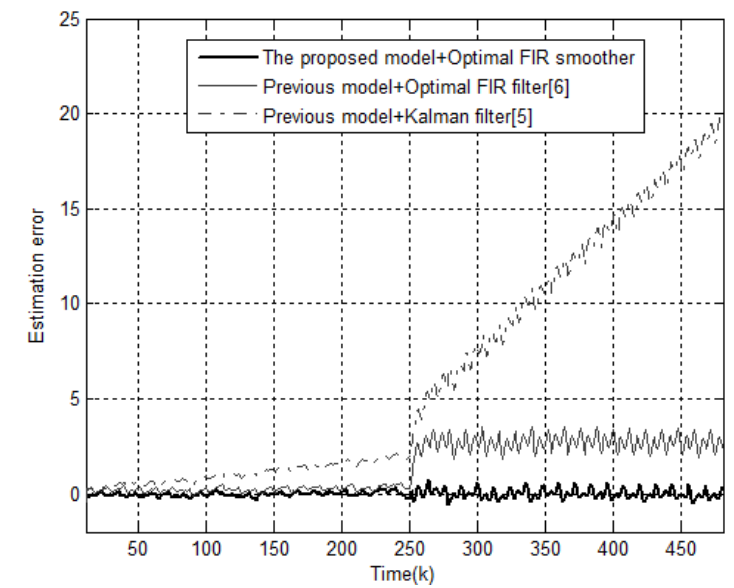

Fig. 1. Estimation errors $\left(c_{k}\right)$ of the previous approaches [5], [6] and the proposed approach

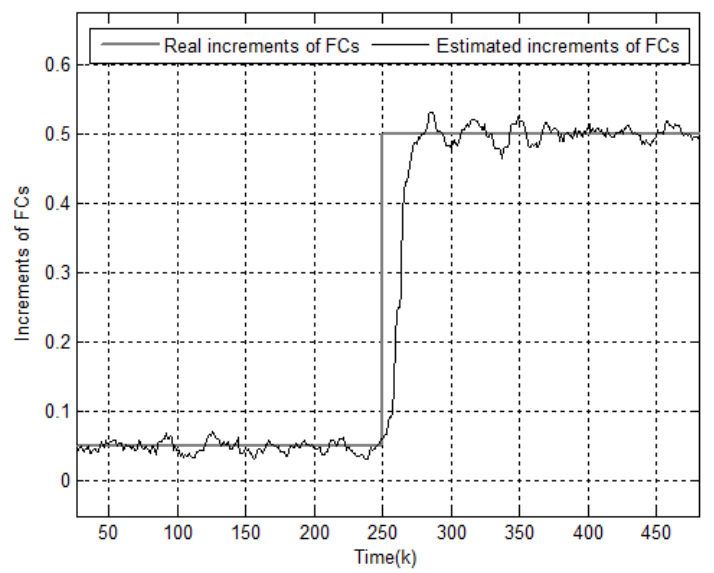

Fig. 2. Estimates of increments of FC at $\pi / 6$

To show the tracking performance of the proposed method, the estimated increments of FCs by using the proposed method are compared with the real increments of FCs in Fig. 2.

As seen in this figure, the estimated increments of FC is closed and tracked well to the real increments of FCs. Thus, it can be said that the proposed scheme can allow the biased random walk models in real situations.

In order to show the benefit of using the proposed new state space model and the optimal fixed-lag FIR smoother, the estimation errors of estimators with the proposed new state space model are compared in Fig. 3.

By comparing the results of optimal FIR filter in Fig. 1 and Fig. 3, it is easily observed that the unbiasedness of the proposed approach comes from the new state space model. Therefore, it is said that the proposed new model could better describe the real quasi-periodic signal than the previous model in [6]. Moreover, it is also shown that the optimal fixed-lag smoother has better tracking ability for estimating time-varying FCs compared with Kalman filtering and the optimal FIR filtering approaches.

The fixed-lag lag size of the optimal fixed-lag FIR

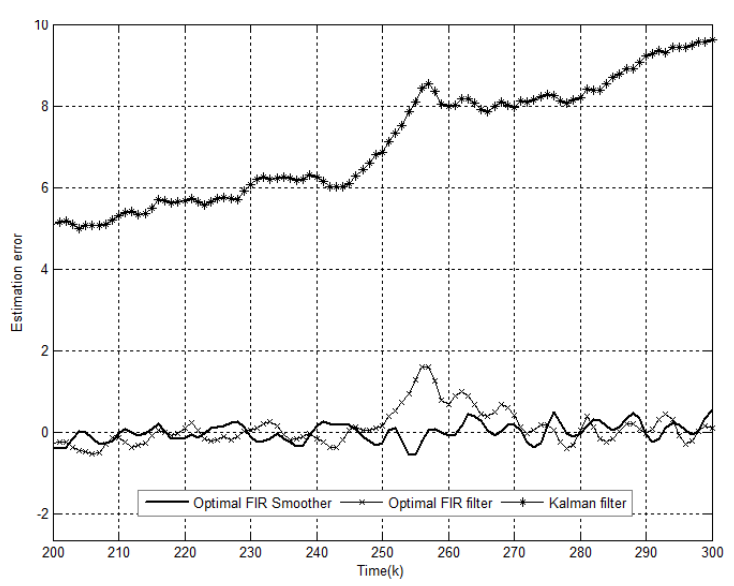

Fig. 3. Estimation errors $\left(c_{k}\right)$ of estimators for the proposed state space model

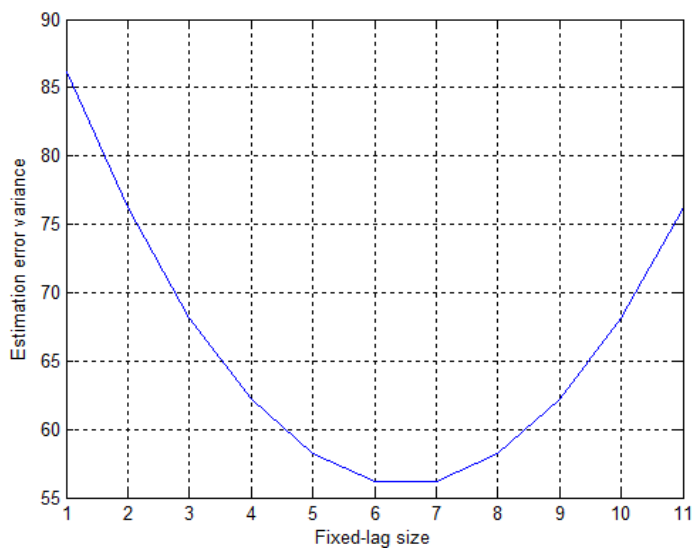

Fig. 4. Estimation error variances for the fixed-lag size

smoother can be considered as a design parameter of estimator. In order to obtain the optimal fixed-lag size of the optimal fixed-lag FIR smoother, estimation error variances are compared with each fixed-lag size in Fig. 4.

By observing Fig. 4, we can easily see that the optimal lag size which is chosen to minimize the estimation error variance is the half of the horizon size. It means that the information which consists of half of the future information and half of the past information estimates better than the other combination of future and past information.

\section{Conclusion}

In this paper, a short-time Fourier analysis method for the quasi-periodic signals with time-varying FCs was proposed by using the optimal fixed-lag FIR smoother. Moreover, a new state model was proposed to represent the noisy quasi-periodic signals composed of harmonics which have time varying FCs instead of using the Gaussian random process for the increments of FCs. In the new state 
model, the increments of the time varying FCs were considered as an auxiliary state and estimated. The optimal fixed-lag FIR smoother applied to the short time Fourier analysis does not require any heuristic approaches such as infinite covariance of the initial state. Moreover, the optimal FIR smoother can be more general and give better estimate than any existing FIR filter. Therefore, the proposed short time Fourier analysis method could give more general solution and better estimate than that of optimal FIR filtering approach. By numerical example, it was shown that the proposed method has better tracking ability for time-varying FCs and more noise-suppression ability than other existing approaches. Moreover, the characteristics of the best lag size for the state model was analyzed by numerical analysis.

\section{Acknowledgements}

This material is based upon work supported by the Ministry of Trade, Industry \& Energy(MOTIE, Korea) under Industrial Technology Innovation Program. No. 10062312 .

\section{References}

[1] J. B. Allen and L.R. Rabiner, "A unified approach to short-time Fourier analysis and synthesis," Proc. IEEE. vol. 65, Nov, 1977.

[2] F. J. Harris, "On the use of windows for harmonic analysis with the discrete Fourier transform," Proc., IEEE, vol. 66, Jan., 1978.

[3] G. H. Hostetter, "Recursive discrete Fourier transformation," IEEE Trans. Acoust., Speech, Signal Processing, vol. ASSP-28, Apr., 1980.

[4] R. R. Bitmead, "On recursive discrete Fourier transformation," IEEE Trans. Acoust., Speech, Signal Processing, vol. ASSP-30, Apr., 1982.

[5] R. R. Bitmead, A. C. Tsoi, and P. J. Parker "A Kalman filtering approach to short-time Fourier analysis," IEEE Trans. Acoust., Speech, Signal Processing, vol. ASSP-34, Dec., 1986.

[6] S. H. Park, W. H. Kwon, O. K. Kwon, and M. J. Kim, "Short-time Fourier analysis via optimal harmonic FIR filters," IEEE Trans. Signal Processing, vol. 45, no. 6, June, 1997.

[7] B. K. Kwon, S. Han, O. K. Kwon, and W. H. Kwon, "Minimum variance FIR Smoother for Discrete-time systems," IEEE Signal Processing Letters, vol. 14, no. 8, Aug., 2007.

[8] Z. Quan, S. Han, J. H. Park, and W. H. Kwon, "Robust FIR filters for linear continuous-time state space models with uncertainties," IEEE Signal Processing Letters, vol. 15, Oct., 2008.
[9] W. H. Kwon and S. Han, "Receding Horizon Control," Springer, 2005.

[10] S. H. Han, W. H. Kwon, and P. S. Kim, "Receding horizon unbiased FIR filters for continuous-time state-space models without a priori initial state information," IEEE Trans. Automatic Control, vol. 46, no. 5, May, 2001.

[11] W. H. Kwon, P. S. Kim, and S. H. Han, "A receding horizon unbiased FIR filter for discrete-time state space models," Automatica, vol. 38, no. 5, May, 2002.

[12] H. H. Giv, "Directional short-time Fourier transform," Journal of Mathematical Analysis and Applications, vol. 399, no. 1, pp. 100-107, March, 2013.

[13] L. Durak, "Shift-invariance of short-time Fourier transform in fractional Fourier domains," Journal of Franklin Institute, vol. 346, no. 2, pp. 136-146, March, 2009.

[14] C. Fernandez and A. Galbis, "Annihilating sets for the short time Fourier transform," Advances in Mathematics, vol. 224, no. 5, pp. 1904-1926, Aug., 2010.

[15] R. Frein and S. T. Rickard, "The Synchronized ShortTime-Fourier-Transform: Properties and Definitions for Multichannel Source Separation," IEEE Trans. Signal Processing, vol. 59, no. 1, pp. 91-103, Jan, 2011.

[16] S.-W. Kim, S.-Y. Kim, and S. Nam, "Short-Time Fourier Transform of Deeply Located Tunnel Signatures Measured by Cross-Borehole Pulse Radar," IEEE Geoscience and Remote Sensing Letters, vol. 8, no. 3, pp. 493-496, March, 2011.

[17] H. Hajimolahoseini, M. R. Taban, and H. SoltanianZadeh, "Extended Kalman Filter frequency tracker for nonstationary harmonic signals," Measurement, vol. 45, no. 1, pp. 126-132, Jan, 2012.

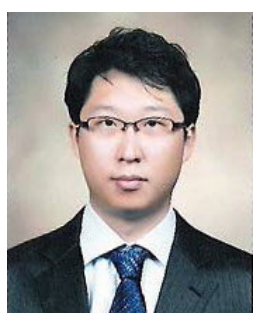

Bo-Kyu Kwon He received his B.S. degree in electronic engineering from Inha University, Korea in 2002. He received the M.S. and Ph.D. degrees in School of electrical engineering and computer science from Seoul National University (SNU) in 2005 and 2008, respectively. For 2002-2003, he was a researcher at the Engineering Research Center for Advanced Control and Instrumentation of SNU. Also, he was with Samsung Heavy Industries as a Senior Research Engineer from 2008 to 2010. Since 2010, he has been with the Department of Control and Instrumentation Engineering, Kangwon National University, Korea. His main research interests are in the areas of estimation and control theory, CACSD(Computer Aided Control System Design) Tool, remote control system, robotics and robot control algorithm. 


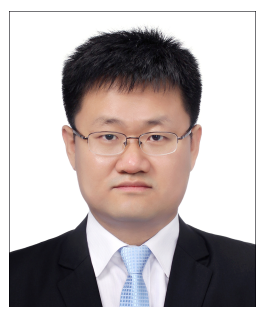

Soohee Han He received the B.S. degree in electrical engineering from Seoul National University (SNU), Seoul, Korea in 1998. He received the M.S. and Ph.D. degrees in School of electrical engineering and computer science from SNU in 2000 and 2003, respectively. From 2003 to 2007, he was a researcher at the Engineering Research Center for Advanced Control and Instrumentation of SNU. In 2008, he was a senior researcher at the robot $\mathrm{S} / \mathrm{W}$ research center. From 2009 to 2014, he was with the Department of Electrical Engineering, Konkuk University, Seoul, Korea. Since 2014, he has been with the Department of Creative IT Engineering, POSTECH, Pohang, Korea. His main research interests are in the areas of computer aided control system design, distributed control system, time delay system, and stochastic signal processing. He is currently an Associated Editor of International Journal of Control, Automation, and Systems, and Journal of Electrical Engineering \& Technology. In 2012, he was a Guest Editor of Mathematical Problems in Engineering for optimal control problems in engineering. He served as the financial chair of IFAC and ICCAS for 2008 and 2012, respectively.

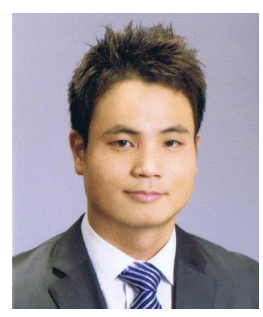

Sekyung Han He received his B.S. degree in electrical engineering and computer science from Hanyang University, Seoul, Korea in 2002, his M.S. in School of electrical engineering and computer science from Seoul National University (SNU), Seoul, Korea in 2007, and $\mathrm{PhD}$ from the department of information and science technology from the University of Tokyo, Japan in 2012, respectively. In SK Innovation Co. LTD., he was involved in the development of the battery system for electric vehicles. Specifically, he was engaged in the development of the battery management system. Currently, he is an assistant professor in Kyungpook National University in Korea and has interests in the implementation of smart distribution system utilizing an energy management system with energy storage. 